\title{
Erratum: "Efficient and Repetitive Neutron Generation by Double-Laser-Pulse Driven Photonuclear Reaction" [Plasma Fusion Res. 13, 2404009 (2018)]
}

Yasunobu ARIKAWA, Yusuke KATO, Yuki ABE, Shuto MATSUBARA, Hidetaka KISHIMOTO, Nozomi NAKAJIMA, Alessio MORACE, Akifumi YOGO, Hiroaki NISHIMURA, Mitsuo NAKAI, Shinsuke FUJIOKA, Hiroshi AZECHI, Kunioki MIMA ${ }^{1)}$, Shunsuke INOUE ${ }^{2)}$, Yoshihide NAKAMIYA ${ }^{2)}$, Kensuke TERAMOTO ${ }^{2)}$, Masaki HASHIDA ${ }^{2)}$ and Shuji SAKABE ${ }^{2)}$ Institute of Laser Engineering, Osaka University, 2-6 Yamadaoka, Suita, Osaka 565-0871, Japan

1) The Graduate School for the Creation of New Photonics Industries, 1955-1 Kurematsu-cho, Nishi-ku, Hamamatsu, Shizuoka 431-1202, Japan

2) Advanced Research Center for Beam Science, Institute for Chemical Research, Kyoto University, Gokasho, Uji, Kyoto 611-0011, Japan

(Received 23 February 2018 / Accepted 26 February 2018)

(c) 2018 The Japan Society of Plasma Science and Nuclear Fusion Research

DOI: $10.1585 /$ pfr. 13.2904019

"The Collaborative Research Program of Institute for Chemical Research, Kyoto University (grant \#20175)" was missing in Acknowledgment. Acknowledgment should be as follows;

This work is supported by the Collaborative Research Program of Institute for Chemical Research, Kyoto University (grant \#2017-5), Adaptable Seamless Technology transfer Program through target driven R\&D (A-STEP) of Japan Science and Technology Agency, Promotion of Science under the contracts of Grant-in-Aids for Scientific Research [Nos. 24244095, 2624043, 23360413, 16H02245], for Young Scientists [Nos. 24686103, 24684044], for Challenging Exploratory Research [Nos. 25630419, 16K13918], for the JSPS fellows [No. 14J06592], and the auspices of the Japanese Ministry of Education, Culture, Sports, Science and Technology (MEXT) project on "Promotion of relativistic nuclear physics with ultraintense laser", and NIFS collaboration research program [NIFS12KUGK057, NIFS12KUGK070, NIFS11KUGK 053, NIFS15KUGK087]. 